\title{
“Inject-Mix-React-Separate-and-Quantitate" (IMReSQ) Method for Screening Enzyme Inhibitors
}

\author{
Edmund Wong, ${ }^{\dagger}$ Victor Okhonin, ${ }^{\dagger}$ Maxim V. Berezovski, ${ }^{\dagger}$ Tomoyoshi Nozaki, ${ }^{\ddagger}$ Herbert Waldmann, ${ }^{\S}$ \\ Kirill Alexandrov," and Sergey N. Krylov ${ }^{*, \dagger}$ \\ Department of Chemistry, York University, Toronto, Ontario M3J 1P3, Canada, Department of Parasitology, \\ National Institute of Infectious Diseases, Tokyo 162-8640, Japan, Department of Chemical Biology and Department \\ of Physical Biochemistry, Max Planck Institute of Molecular Physiology, 44227 Dortmund, Germany
}

Received June 15, 2008; E-mail: skrylov@yorku.ca

Many enzymes involved in regulatory cellular processes are considered attractive therapeutic targets and their inhibitors are potential drug candidates. ${ }^{1}$ Screening of combinatorial libraries for enzyme inhibitors is pivotal to identifying hit compounds for the development of enzyme-targeting drugs. Here we introduce the first method for screening enzyme inhibitors which is applicable to regulatory enzymes and consumes only nanoliter volumes of the reactant solutions. We name the method inject-mix-react-separate-and-quantitate (IMReSQ). The concept of the method is shown in Figure 1. First, nanoliter volumes of substrate, candidate inhibitor, and enzyme solutions are injected separately (from microliter volumes in cupped vials) by pressure into a capillary as separate plugs without the need of nanoliterscale liquid handlers. Second, the plugs are mixed inside this capillary microreactor by transverse diffusion of laminar flow profiles (TDLFP). ${ }^{2}$ Third, the reaction mixture is incubated to form the enzymatic product. Fourth, the product is separated from the substrate inside the capillary by electrophoresis. Fifth, the amounts of the product and substrate are quantitated. In this proof-of-principle work, we applied the method to study inhibition of recently cloned protein farnesyltransferase (FT) from parasite Entamoeba histolytica (Eh); this enzyme is a potential therapeutic target for antiparasitic drugs. ${ }^{3,4} \mathrm{We}$ identified three previously unknown inhibitors of EhFT and proved that IMReSQ could be used for accurately ranking the potencies of inhibitors.

Methods for screening enzyme inhibitors can be divided into two broad categories: homogeneous, which monitor product formation without its physical separation from the substrate, and separation-based, which separate the product from the substrate by means of chromatography or electrophoresis prior to its quantitation. Recent advances in printing chemical libraries made it possible to transfer homogeneous methods from microtiter plates to microarrays, which require only nanoliter volumes of reagents. ${ }^{5}$ Microarrays, however, require substrates that do not fluoresce before being converted into fluorescent products. Such fluorogenic substrates are not available for the majority of regulatory enzymes, for example, prenyltransferases, glycosyltransferases, and kinases. In separation-based methods, simple fluorescently labeled substrates can be used instead of fluorogenic substrates. Fluorescently labeled substrates are available for many regulatory enzymes. ${ }^{6}$ If separation is carried out in a narrow-bore capillary, only nanoliter volumes of reaction mixtures are consumed. Because of the lack of a generic way of mixing solutions inside the capillary, however, the reaction mixture must be prepared in a vial outside the capillary with a volume of at least several microliters. This work was inspired by the insight that TDLFP can be used to mix nanoliter volumes of enzyme, substrate, and candidate inhibitor, injected into the capillary as separate plugs. Thus far, TDLFP had been used to mix two reactants

\footnotetext{
† York University.

* National Institute of Infectious Diseases.

$\$$ Department of Chemical Biology, Max Planck Institute of Molecular Physiology.

" Department of Physical Biochemistry, Max Planck Institute of Molecular Physiology.
}

only. ${ }^{2}$ Here, we demonstrate the TDLFP-based mixing of four reactants-enzyme, two substrates, and inhibitor-followed by enzymatic product formation, separation of the product from the substrate, and quantitation of the formed product and remaining substrate.

The EhFT enzyme was a recombinant protein produced as described elsewhere. ${ }^{4}$ The enzyme transfers the farnesyl group from farnesyl pyrophosphate (substrate $1, \mathrm{~S} 1$ ) to a fluorescently labeled pentapeptide (substrate 2, S2), which mimics Ras protein, its native substrate. The farnesylated pentapeptide (product, P) was separated from S2 by capillary electrophoresis and fluorescent detection was used to quantitate the amounts of P and S2. Farnesyltransferase inhibitors (FTIs) tested in this work were commercial FTI-276 and FTI-277, previously shown to inhibit $E h \mathrm{FT}^{4}$ and human FT, ${ }^{7}$ respectively, as well as new compounds, FTI-343, FTI-391, FTI651 , and FTI-656, recently proven to inhibit mammalian $\mathrm{FT}^{8}$ but never tested for EhFT. Fluorescence detection was used in this work; however, other detection approaches, such as light absorption and mass-spectrometry, can also be used in IMReSQ.

First, we studied TDLFP mixing of the four reaction components using computer simulation (see the Supporting Information). An algorithm for the optimization of the plug order in TDLFP mixing has not been developed yet; therefore, strictly speaking, we could not optimize the plug order. We could, however, numerically simulate the concentration profiles of the mixed reaction components along the capillary for any given order of plugs (Figure 2A). Using such a simulation we tested several plug orders that seemed reasonable on the basis of two simple criteria: the number of plugs had to be small while the spatial overlap of the components after mixing had to be significant. The plug order chosen for further work was: S1, S2, enzyme (E), inhibitor (I), and S1 again (Figure 2A). Plugs of an enzymatic buffer were injected before and after injecting the reaction components to (i) isolate the reaction mixture from an electrophoresis buffer containing a surfactant and (ii) improve the quality of mixing. The simulated after-mixing concentration profiles of the four components did not overlap perfectly; however, they revealed a significant reaction zone with all four components present (Figure 2A). There was also a reaction zone with $\mathrm{S} 1, \mathrm{~S} 2$, and $\mathrm{E}$, but without I, suggesting that complete inhibition with this mixing scenario was not achievable.

In TDLFP, the required mixing time is defined by the time of transverse diffusion of the largest molecule, EhFT. Our computer simulation showed that for the experimental conditions used, the sufficient mixing time was less than $1 \mathrm{~min}$. The reaction time was longer than the mixing time, which suggested that only a negligible amount of the product was formed during mixing.

Second, we experimentally demonstrated that TDLFP indeed mixed the four reaction components and that the product formation could be observed along with the inhibition. The absence of either of the components meant that the concentration of this component in the injected plug was zero (an enzyme buffer was injected). In 


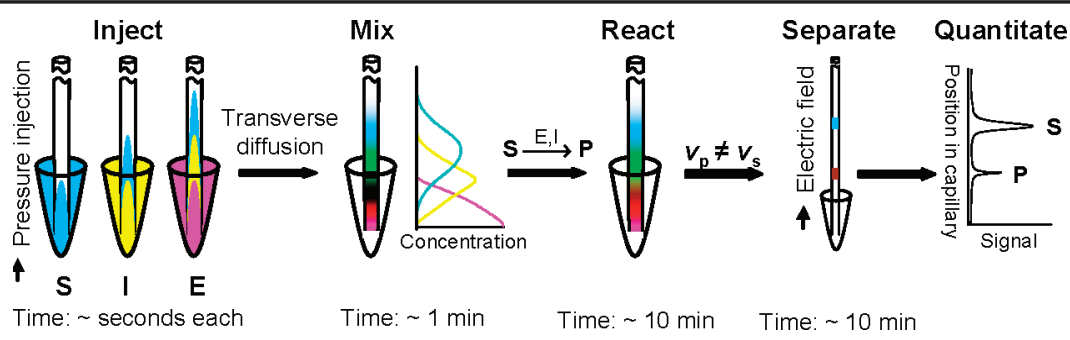

Figure 1. The concept of IMReSQ. The reaction components (substrate, S; inhibitor, I; and enzyme, E) are injected into the capillary as separate plugs, mixed by TDLFP, reacted to form the product $(\mathrm{P})$, and separated. $\mathrm{S}$ and $\mathrm{P}$ are quantitated after separation.
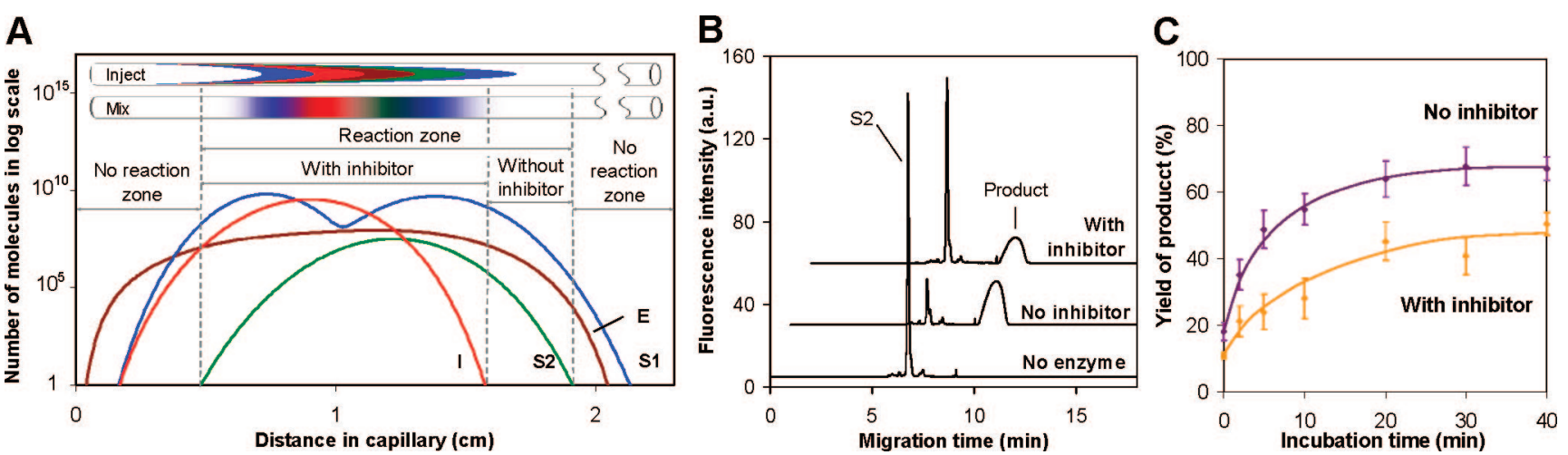

Figure 2. IMReSQ for inhibition of EhFT enzyme. (A) Simulated distribution of reaction components in the capillary after injection (top) and after mixing (bottom) for the following plug order: enzymatic buffer (white), FPP (S1, blue), fluorescent peptapeptide substrate (S2, green), EhFT enzyme (E, brown), inhibitor (I, red), FPP again, and enzymatic buffer again. (B) Experimental result of IMReSQ analysis of EhFT inhibition by FTI-276 after 40-min incubation following injection and mixing of reaction components depicted in panel A. The preinjection concentrations were $75 \mu \mathrm{M}$ for $\mathrm{S} 1,0.2 \mu \mathrm{M}$ for $\mathrm{S} 2,3 \mu \mathrm{M}$ for $\mathrm{E}$, and $50 \mu \mathrm{M}$ for I. (C) Kinetics of product formation with and without inhibitor (50 $\mu \mathrm{M}$ FTI-276).

this part of our study, we used the FTI-276 inhibitor that had been proven to inhibit EhFT. ${ }^{4}$ When the concentration of E was zero, no $\mathrm{P}$ was formed and a single peak of $\mathrm{S} 2$ was detected (Figure 2B, lower trace). In the presence of $\mathrm{E}$ but without I, S2 was converted in to $\mathrm{P}$ and, accordingly, two peaks were observed after $\mathrm{P}$ was separated from the remaining S2 (Figure 2B, middle trace). Finally, in the presence of $\mathrm{I}$, the reaction rate was lower and the amount of $\mathrm{P}$ formed during the same incubation time was smaller. The peak of $\mathrm{P}$ was smaller while the peak of the remaining S2 was higher (Figure 2B, upper trace) than those in the absence of I. When the incubation time varied, the reaction kinetics could be measured. The kinetics in the absence and presence of I were classical Michaelis kinetics (Figure 2C). Thus, all four components were mixed to the level at which the rate of $\mathrm{P}$ formation depended on $\mathrm{I}$.

Third, we tested if the IMReSQ method could be used to quantitatively rank the potencies of inhibitors. Conventionally, $\mathrm{IC}_{50}$ values (inhibitor concentrations that cause $50 \%$ reduction of the reaction rate) are used to rank inhibitors. $\mathrm{IC}_{50}$ depends on enzyme and substrate concentrations and, thus, inapplicable to IMReSQ, in which the solutions are not ideally mixed. To quantitatively rank inhibitors by IMReSQ, we suggest PIC $_{50}$, which is a preinjection inhibitor concentration that causes $50 \%$ reduction in the reaction rate. We compared ranking of several potential inhibitors of EhFT by a "traditional" method using $\mathrm{IC}_{50}$ and by the IMReSQ method using $\mathrm{PIC}_{50}$. In the traditional method, microliter volumes of S1, S2, I, and E were mixed in a vial and incubated to form P. A nanoliter volume of the reaction mixture was injected into the capillary and $\mathrm{P}$ was separated from remaining S2 and their amounts were quantitated. The $\mathrm{PIC}_{50}$ to $\mathrm{IC}_{50}$ ratio was identical within the error limits (Table 1). The constant ratio suggests that IMReSQ can be used for quantitatively ranking potencies of the inhibitors. Four inhibitors of mammalian farnesyltransferase (FTI-276, FTI-277, FTI-651, and FTI-656) were found to be potent for $E h \mathrm{FT}$ and can, therefore, be used as hit compounds for drug development. To conclude, IMReSQ is the first method that consumes
Table 1. $\mathrm{IC}_{50}$ and $\mathrm{PIC}_{50}$ for Inhibition of EhFT Determined by Traditional (in-Vial Reaction) and IMReSQ Methods, Respectively

\begin{tabular}{cccr}
\hline candidate inhibitor & traditional IC $(u \mathrm{M})$ & $\mathrm{IMReSQ}_{5 \mathrm{PI}}(\mu \mathrm{M})$ & \multicolumn{1}{c}{$\mathrm{PIC}_{50} / \mathrm{IC}_{50}$} \\
\hline FTI-276 & $1.1 \pm 0.3$ & $11.2 \pm 3.2$ & $10.2 \pm 2.9$ \\
FTI-277 & $2.1 \pm 0.4$ & $18.9 \pm 3.8$ & $9.0 \pm 1.8$ \\
FTI-343 & not measurable & not measurable & \\
FTI-591 & not measurable & not measurable & \\
FTI-651 & $71 \pm 17$ & $800 \pm 70$ & $11.3 \pm 2.7$ \\
FTI-656 & $61 \pm 18$ & $620 \pm 90$ & $10.2 \pm 3.1$
\end{tabular}

nanoliter volumes of the reaction components per analysis and is applicable to regulatory enzymes. IMReSQ can be used with commercially available single-capillary instrumentation for screening small libraries. Screening large libraries with IMReSQ will require specialized multicapillary instrumentation.

Acknowledgment. The work was funded by NSERC Canada.

Supporting Information Available: Supporting materials and methods. This material is available free of charge via the Internet at http:// pubs.acs.org.

\section{References}

(1) Sen, N.; Majumder, H. K. Curr. Pharm. Des. 2008, 14, 839-846.

(2) Okhonin, V.; Liu, X.; Krylov, S. N. Anal. Chem. 2005, 77, 5925-5929.

(3) Ali, V.; Nozaki, T. Clin. Microbiol. Rev. 2007, 20, 164-187.

(4) Kumagai, M.; Makioka, A.; Takeuchi, T.; Nozaki, T. J. Biol. Chem. 2004, 279, 2316-2323.

(5) Gosalia, D. N.; Diamond, S. L. Proc. Natl. Acad. Sci. U.S.A. 2003, 100, 8721-8726.

(6) Berezovski, M.; Li, W.-P.; Poulter, C. D.; Krylov, S. N. Electrophoresis 2002, 23, 3398-3403.

(7) Lerner, E. C.; Qian, Y.; Blaskovich, M. A.; Fossum, R. D.; Vogt, A.; Sun, J.; Cox, A. D.; Der, C. J.; Hamilton, A. D.; Sebti, S. M. J. Biol. Chem. 1995, 270, 26802-26806.

(8) Dursina, B.; Reents, R.; Delon, C.; Wu, Y.; Kulharia, M.; Thutewohl, M.; Veligodsky, A.; Kalinin, A.; Evstifeev, V.; Ciobanu, D.; Szedlacsek, S. E.; Waldmann, H.; Goody, R. S.; Alexandrov, K. J. Am. Chem. Soc. 2006, 128, 2822-2835.

JA804544X 


\title{
SUPPORTING INFORMATION
}

\section{“Inject-Mix-React-Separate-and-Quantitate” (IMReSQ) Method for Screening of Enzyme Inhibitors}

\author{
Edmund Wong, ${ }^{1}$ Victor Okhonin, ${ }^{1}$ Maxim V. Berezovski, ${ }^{1}$ Tomoyoshi Nozaki, ${ }^{2}$ Herbert \\ Waldmann, ${ }^{3}$ Kirill Alexandrov, ${ }^{4}$ and Sergey N. Krylov ${ }^{1 *}$ \\ ${ }^{1}$ Department of Chemistry, York University, Toronto, Ontario M3J 1P3, Canada \\ ${ }^{2}$ Department of Parasitology, National Institute of Infectious Diseases, Tokyo 162-8640, Japan \\ ${ }^{3}$ Department of Chemical Biology, Max Planck Institute of Molecular Physiology, 44227 \\ Dortmund, Germany \\ ${ }^{4}$ Department of Physical Biochemistry, Max Planck Institute of Molecular Physiology, 44227 \\ Dortmund, Germany
}

\section{Supporting Materials and Methods}

Materials. Farnesyl pyrophosphate (FPP) and FTI-276 were purchased from Sigma-Aldrich (Oakville, ON, Canada). FTI-277 was purchased from Calbiochem (San Diego, CA, USA). Fluorescently-labeled pentapeptide - 2',7'-difluorofluorescein-5-carboxyl-glycinyl-cysteinylvalinyl-isoleucinyl-alanine - was purchased from AnaSpec (San Jose, CA, USA). Recombinant protein farnesyltransferase from enteric protozoan parasite Entamoeba histolytica (EhFT) was expressed in Escherichia coli and purified as described elsehwhere. ${ }^{1}$ FTI-343, FTI-591, FTI-651 and FTI-656 were synthesized in a parallel approach and isolated as pure compounds as described elsewhere. ${ }^{2}$ All chemicals were purchased from Sigma-Aldrich, Caledon, or BDH (Toronto, ON, Canada). An uncoated fused-silica capillary was purchased from Polymicro (Phoenix, AZ, USA). All solutions were made using Milli-Q-quality deionized water filtered through a $0.22 \mu \mathrm{m}$ filter (Millipore, Nepean, ON, Canada).

EhFT-catalyzed farnesylation. EhFT catalyzes the transfer of the farnesyl group from FPP (substrate 1) to the cysteine residue of the fluorescently-labeled pentapeptide (substrate 2) that mimics Ras protein, the native substrate of EhFT (Fig. S1). Farnesylation can be inhibited by peptidomimetic compounds. In this study, commercial FTI-276 and FTI-277, as well as new compounds, FTI-343, FTI-591, FTI-651 and FTI-656, were used (Fig. S2). 


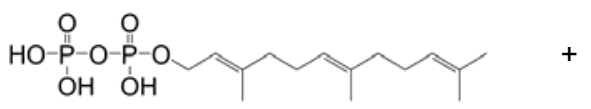

Farnesyl pyrophosphate (FPP, S1)

$\mathrm{MW}=382 \mathrm{~g} / \mathrm{mol}$ $\mathrm{D}=3.5 \times 10^{-6} \mathrm{~cm}^{2} / \mathrm{s}$

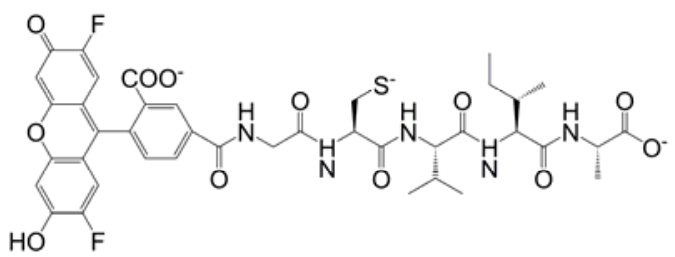

Fluorescently-labeled pentapeptide (S2)

$$
\mathrm{MW}=852 \mathrm{~g} / \mathrm{mol}
$$$$
\mathrm{D}=2.1 \times 10^{-6} \mathrm{~cm}^{2} / \mathrm{s}
$$

EhFT (E)

$\mathrm{MW}=78.2 \mathrm{kDa}$

$\mathrm{D}=5.6 \times 10^{-7} \mathrm{~cm}^{2} / \mathrm{s}$

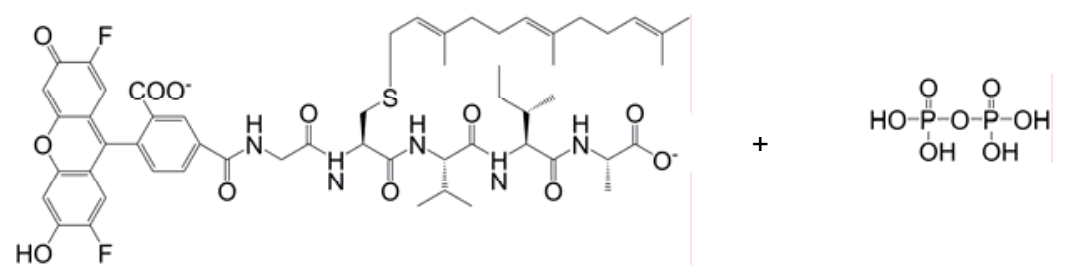

Farnesylated product $(\mathrm{P})$

Figure S1. Farnesylation of fluorescently-labeled pentapeptide catalyzed by EhFT. The figure also contains molecular weights and diffusion coefficients of the reactants.<smiles>CSCCC(NC(=O)c1ccc(NCC(N)CS)cc1-c1ccccc1)C(=O)O</smiles>

FTI-276

$\mathrm{MW}=433 \mathrm{~g} / \mathrm{mol}$

$\mathrm{D}=3.3 \times 10^{-6} \mathrm{~cm}^{2} / \mathrm{s}$

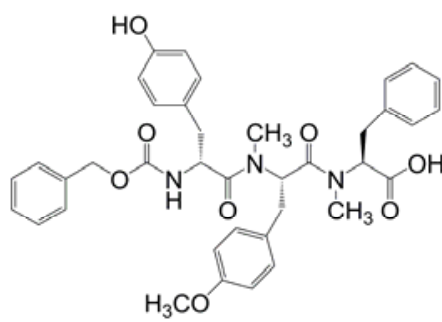

FTI-591

$\mathrm{MW}=668 \mathrm{~g} / \mathrm{mol}$

$\mathrm{D}=2.4 \times 10^{-6} \mathrm{~cm}^{2} / \mathrm{s}$

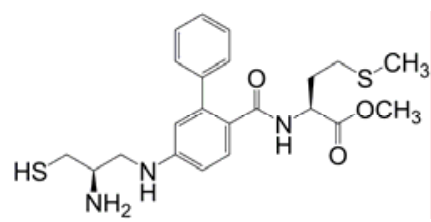

FTI-277

$\mathrm{MW}=334 \mathrm{~g} / \mathrm{mol}$

$\mathrm{D}=3.9 \times 10^{-6} \mathrm{~cm}^{2} / \mathrm{s}$

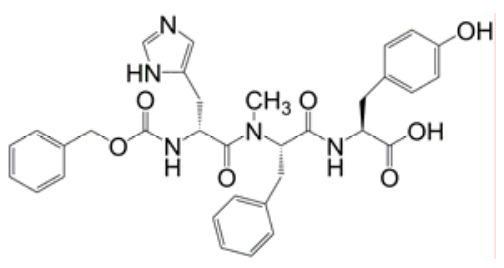

FTI-651

$\mathrm{MW}=614 \mathrm{~g} / \mathrm{mol}$

$\mathrm{D}=2.5 \times 10^{-6} \mathrm{~cm}^{2} / \mathrm{s}$

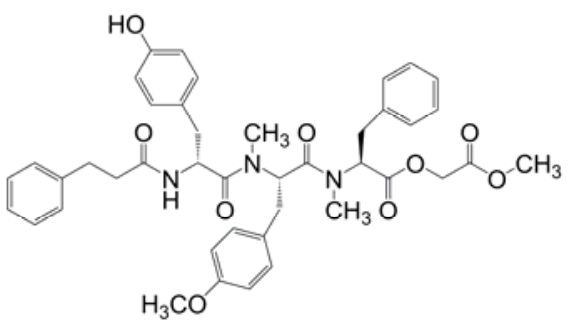

FTI-343

$\mathrm{MW}=734 \mathrm{~g} / \mathrm{mol}$

$\mathrm{D}=2.3 \times 10^{-6} \mathrm{~cm}^{2} / \mathrm{s}$

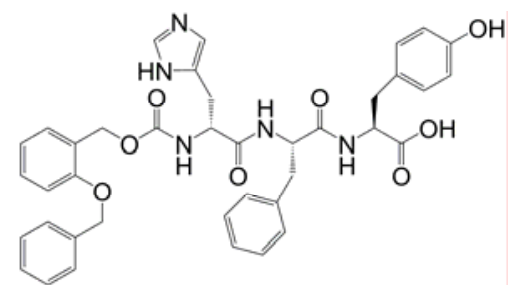

FTI-656

$\mathrm{MW}=705 \mathrm{~g} / \mathrm{mol}$

$\mathrm{D}=2.3 \times 10^{-6} \mathrm{~cm}^{2} / \mathrm{s}$

Figure S2. Structures, molecular weights, and diffusion coefficients of inhibitors. 
Numerical simulation. The mathematical model to simulate the mixing of reaction components calculates the concentrations of substances mixed by TDLFP as functions of the position in the capillary and time passed since the beginning of mixing. By neglecting the mixing by longitudinal diffusion, we obtained the simplified equation for the mathematical model. The algorithm of numerical simulation assumed that the shape of the injected plug was parabolic if the diffusion coefficient was zero. If the diffusion coefficient was not zero, the shape was distorted by transverse diffusion. To calculate the distorted shape, we used a two-sub-iteration computational procedure, which modeled: (i) injection without diffusion and (ii) diffusion without injection. The first sub-iteration was designed so that after a given number of iterations, the error of modeling associated with using a finite grid was averaged out. In the second subiteration, a backward Euler method with triagonal matrix algorithm was used. The program input parameters are: diffusion coefficients and concentrations of all components, capillary inner radius, velocity and time of injection for every component, as well as the order of injected plugs. The computer program can be found at www.chem.yorku.ca/profs/krylov in the Research section.

Diffusion coefficients used for computer simulations were estimated by the Mark-HouwinkSacurada equation ${ }^{3}$ (constants required in the equation were determined using published known diffusion coefficients ${ }^{4-5}$ ) and presented in Figs. S1 and S2 above.

Instrumentation. An uncoated fused-silica capillary with a length of $50 \mathrm{~cm}(40 \mathrm{~cm}$ to the detection window) and inner and outer diameters of 75 and $365 \mu \mathrm{m}$, respectively, was used in all experiments. The capillary was mounted on a capillary electrophoresis (CE) instrument (P/ACE MDQ, Beckman Coulter, Fullerton, CA, USA), which could facilitate automated injection of solutions by pressure. The reaction product $(\mathrm{P})$ and unreacted substrate (S2) were separated by $\mathrm{CE}$ and quantitated with laser-induced fluorescence (LIF) detection (fluorescence excitation at $488 \mathrm{~nm}$ and fluorescence detection at $520 \mathrm{~nm}$ ).

IMReQ procedure. The reactants were injected into the capillary in the following order: enzymatic buffer ( $50 \mathrm{mM}$ Tris-HCl, $10 \mathrm{mM} \mathrm{MgCl}, 10 \mu \mathrm{M} \mathrm{ZnCl}_{2}, 5 \mathrm{mM}$ DTT, pH 7.1) (0.2 psi, $5 \mathrm{~s}$ ), substrate 1 ( $0.2 \mathrm{psi}, 5 \mathrm{~s}$ ), substrate 2 ( $0.2 \mathrm{psi}, 5 \mathrm{~s}$ ), EhFT enzyme ( $0.2 \mathrm{psi}, 5 \mathrm{~s})$, inhibitor or enzymatic buffer ( $0.2 \mathrm{psi}, 5 \mathrm{~s})$, substrate 1 ( $0.2 \mathrm{psi}, 5 \mathrm{~s}$ ), and enzymatic buffer again (0.2 psi, 20 s). The first and the last plugs of enzymatic buffer isolated the reaction zone from an SDScontaining electrophoresis buffer (25 mM borax, 25 mM SDS, pH 9.3). SDS had a dual role. 
First, it improved the quality of separation of substrate 2 and product. Second, SDS helped stop the enzymatic reactions by denaturing the enzyme when electrophoretic separation started. The last plug also played a role in improving the quality of mixing, because it increased the length of the longitudinal interface between the reactant plugs. The reactants were then mixed by TDLFP and allowed to react for a certain time. The reaction was stopped by applying an electric field of $500 \mathrm{~V} / \mathrm{cm}$ to separate the enzyme from the substrates. The product and unreacted substrate 2 were quantitated with LIF by measuring the normalized areas of corresponding peaks (areas of peaks divided by their migration times). The temperature of the capillary was maintained at $25 \pm 0.2{ }^{\circ} \mathrm{C}$. The thermostated storage room of the $\mathrm{CE}$ instrument was maintained at $4{ }^{\circ} \mathrm{C}$ in order to prevent the degradation of reagents during the analyses.

The traditional method. $5 \mu \mathrm{L}$ of the reaction mixture was prepared in a vial and contained 15 to $75 \mu \mathrm{M} \mathrm{S1}, 50 \mathrm{nM} \mathrm{S2}$, and 1.2 to $3 \mu \mathrm{M}$ enzyme in the enzymatic buffer. The reaction was initiated by adding the enzyme. The mixture was vortexed and incubated at $25{ }^{\circ} \mathrm{C}$. To stop the reaction, an equal amount of the SDS-containing electrophoresis buffer was added to the reaction mixture. The resulting solution was injected into the capillary by a pressure pulse of 0.5 psi and 5 s. The product was separated from S2 and both were quantitated with LIF in the same manner as in IMReSQ.

Effect of inhibitor concentration on EhFT activity. Using the IMReSQ method, $30 \mu \mathrm{M}$ S1, $200 \mathrm{nM} \mathrm{S2,} 1.2 \mu \mathrm{M}$ enzyme, and increasing concentrations of an inhibitor were injected into the capillary in the described above order. Using the traditional method, $15 \mu \mathrm{M} \mathrm{S1,} 50 \mathrm{nM} \mathrm{S} 2$, $300 \mathrm{nM}$ enzyme, and increasing concentrations of inhibitor were incubated in a vial. In both IMReSQ and the traditional methods, the mixture was incubated for $3 \mathrm{~min}$. The reaction was then stopped and the mixture was analyzed as described above. The relative activity was the initial rate of the inhibited reaction relative to the initial rate of the reaction without inhibitor. When $\mathrm{IC}_{50}$ or $\mathrm{PIC}_{50}$ exceeded $1 \mathrm{mM}$, we considered them not measurable.

\section{Supporting Information References}

1. Kumagai, M.; Makioka, A.; Takeuchi, T.; Nozaki, T. J. Biol. Chem. 2004, 279, 2316-2323.

2. Dursina, B.; Reents, R., Delon, C.; Wu, Y.; Kulharia, M.; Thutewohl, M.; Veligodsky, A.; Kalinin, A.; Evstifeev, V.; Ciobanu, D.; Szedlacsek, S.E.; Waldmann, H.; Goody, R.S.; Alexandrov, K. J. Am. Chem. Soc. 2006, 128, 2822-2835.

3. C. Tanford, Physical chemistry of macromolecules, John Wiley Press, New York (1961) 
4. Liu, M.; Li, P.; Giddings, J. C. Protein Sci. 1993, 2, 1520-1531.

5. Derrick, T. S.; Larive, C. K. Appl. Spectrosc. 1999, 53, 1595-1600. 